\title{
Inverse Relationship between Tensile Bond Strength and Dimensions of Bonded Area
}

\author{
Nuria I. Escribano, Maria O. Del-Nero, J ose C. de la Macorra \\ Department of Restorative Dentistry, Faculty of Odontology, Complutense University, Plaza Ramon y Cajal s/n, \\ Ciudad Universitaria, 28040 Madrid, Spain
}

Received 16 May 2002; revised 2 December 2002; accepted 4 December 2002

\begin{abstract}
It is a known fact that there is a relationship between magnitude of bonded area and laboratory tensile test results. This relationship has been described for a range of areas between 1 and $10 \mathrm{~mm}^{2}$, in extracted, nonperfused teeth. The aim of this study is to test this relationship in perfused teeth, with bonded areas ranging from 0.7 to $110.9 \mathrm{~mm}^{2}$. Dentin of 92 sound third human molars was exposed and perfused, and three groups of bonded areas (BA) were delimited: small $\left(0.69-1.89 \mathrm{~mm}^{2}\right)$, medium $\left(8.66-19.54 \mathrm{~mm}^{2}\right)$, and large $(58.91-110.86$ $\mathrm{mm}^{2}$ ). Tensile bond strength (TBS) of three adhesive restorative systems was found. The best nonlinear curve estimation was searched (SPSS 9.0) between TBS and BA, for each and all materials. The best estimation was, for all materials, $\mathrm{TBS}=4.17+10.35 / \mathrm{BA}(\boldsymbol{p}<\mathbf{0 . 0 0 0 1})$. (C) 2003 Wiley Periodicals, Inc. J Biomed Mater Res Part B: Appl Biomater 66B(1): 419-24, 2003
\end{abstract}

Keywords: dental adhesives; tensile tests; microtensile tests; perfusion

\section{INTRODUCTION}

A durable bond between the restorative material and tooth structure is of paramount importance for the success of dental restorations. To find the potential of a dentin bonding system, its mechanical resistance is usually measured determining the shear and/or the tensile bond strength. Nevertheless, this evaluation of dental adhesives is controversial, with little or none agreement in bond-strength data among different authors, due to the variations in the techniques employed and to the different conditions under which the tooth substrates are kept and used. Most methods tend to be very empirical, and they are not well developed to validate theoretical analysis and structure-property relationships. ${ }^{1,2}$ As a result, dentin bond-strength data suffer from considerable scatter, when results from distinct authors are compared. ${ }^{3}$

Results of both types of mechanical tests, shear and tensile, depend greatly on surface area, ${ }^{4}$ among other factors. In these tests, adhesive strength is determined by dividing the failure load by the cross-sectional area of the bonded surface. According to Griffith's theory, ${ }^{5}$ it is more probable to find a defect that initiates the fracture in a larger area than in a smaller one, so the apparent tensile strength of a material decreases when the size of the tested area increases. It has been shown ${ }^{12}$ that any existing defect in the interface can

Corrrespondence to: Jose C. de la Macorra, Department of Restorative Dentistry, Faculty of Odontology, Complutense University, Plaza Ramon y Cajal s/n, Ciudad Universitaria, 28040 Madrid, Spain (e-mail: macorra@odon.ucm.es)

(C) 2003 Wiley Periodicals, Inc. propagate. In order to solve these stress propagation problems, mainly associated with large areas, Sano et al. ${ }^{6}$ developed a miniature tensile testing method, the so-called microtensile bond test. The aim of this test was to avoid the scatter in results, and to eliminate the nonuniform stress distribution.

Finite-element analysis (FEA) helps to explain why the small bonding areas tend to fail at higher stresses compared with traditional large surface area tests: ${ }^{7,8}$ The maximum stress that occurred in smaller specimens was lower than the maximum one of the bigger specimens. The specimens with lower maximum stress have a greater chance to survive longer. ${ }^{8}$

The aim of this study is to provide a perspective for data obtained from tensile bond strength tests with the use of different area ranges in perfused teeth. This experimental approach seeks to find a relationship between the tested area size and the tensile bond strength, and to assess that an increase in size always corresponds with a decrease in tensile strength in a wide area ranges.

\section{MATERIALS AND METHODS}

Extracted human third molars $(n=92)$, free of caries and restorations, were preserved in a $70 \%$ ethanol solution within a month after extraction. Roots and occlusal enamel were removed with a diamond bur under abundant water refrigeration. The pulpal chamber was exposed at the furca level, and its contents were carefully removed with cotton pliers. The exposed occlusal dentin and the cut cervical area were lightly polished with 1200-grit sandpaper ( $\mathrm{SiC}$ paper, Struers A/S, 


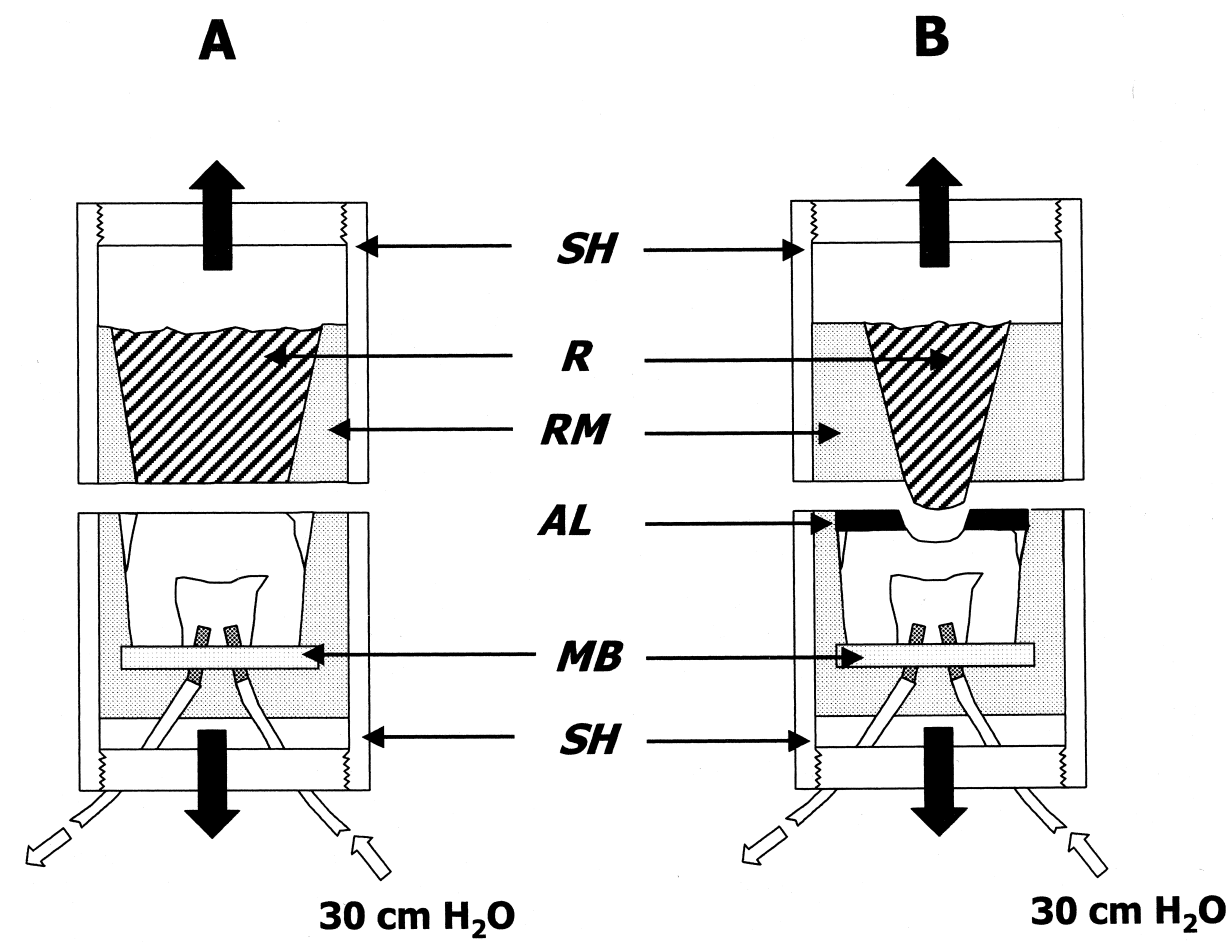

Figure 1. Schematic saggital view of assemblies, after detaching of interface. SH: steel holder, R: restorative material, $\mathrm{RM}$ : embedding resin, $\mathrm{AL}$ : amalgam layer, $\mathrm{MB}$ : methacrylate base.

Copenhagen, Denmark) in a water-cooled polishing machine (Struers Dap-7, Struers, Copenhagen, Denmark). This crown segment was glued to a $1 \times 1-\mathrm{cm}$ methacrylate base with a cyanoacrylate general-purpose adhesive (Super Glue 3, Loctite, Madrid, Spain). This base had two holes where two metal tubes were introduced entering the pulpal chamber, and the passage was sealed (Duralay, Reliance, Dental Mfg. Co., Worth, IL). Lateral areas of the specimen were covered with a nail-polish layer (Revlon, Barcelona, Spain) to reduce leakage.

Specimens were divided in three groups, depending on the size of the area to be tested. In the first group, the entire area of exposed dentin was tested [Figure 1(a)]. These specimens were included in a resin matrix (Cronolita 11700/3115, Plastiform, Madrid, Spain), contained in a steel holder [SH in Figure 1(a)]. Once the embedding resin was cured, dentin and resin surfaces were polished, and a liquid separator (Vaseline) was applied.

Over the entire occlusal cut surface a troncoconical column was made of Plasticine ${ }^{\circledR}$, the upper steel holder was assembled, and a counterpart of same resin was poured in it surrounding the plasticine column. When resin had set, this plasticine was removed, and confronted surfaces of both parts were polished to remove Plasticine and Vaseline residuals. The upper counterpart was intended to act as a matrix for restoration via its central conical perforation, previously containing the Plasticine column.

In the specimens of second and third groups [Figure 1(b)], the occlusal exposed dentin was etched with $37 \%$ ortophosphoric acid (Total Etch, batch B28357, Ivoclar/Vivadent,
Schaan Liechtenstein) for $15 \mathrm{~s}$ and washed thoroughly. Excess water was removed with laboratory absorbent paper. The Scotchbond Multipurpose System (batch 70-2010-1232-8, 3M Dental Products Division, St. Paul, MN) was applied on the dentin surface, to seal it with bonded amalgam (Amalcap Plus Nongamma 2, batch 532288 NN, Vivadent, Liechtenstein), forming a layer of approximately $1 \mathrm{~mm}$ thick. After $24 \mathrm{~h}$, the amalgam surface was polished under water refrigeration. Then, specimens were also included in the lower steel holder with a resin, like specimens in the first group.

The upper steel holder was filled with resin. When this resin had set, it was perforated until the amalgam layer was reached. Then, round expulsive cavities were drilled through amalgam until they reached dentin. In the second group the cavities were drilled with a doughnut bur (diameter $4 \mathrm{~mm}$ ), and in the third group with a round bur (diameter $1 \mathrm{~mm}$ ). The cavities were conditioned (Total Etch, 15 s) to remove the smear layer produced by the bur.

The exposed dentin areas $\left(\mathrm{BA}\right.$, in $\left.\mathrm{mm}^{2}\right)$ of all groups were measured with a stereoscopic microscope and an image analyzer (Leica Qwin Q500 IW, Leica Imaging Systems Ltd., Cambridge, UK).

The perfusion system was a column of $30 \mathrm{~cm}$ of distilled water, connected with a tube to the pulpal chamber through one of the metal catheters. Via the other metal tube, air was extracted from the pulpal chamber until the chamber was filled with distilled water. After that, the tube was sealed. The specimens were perfused $24 \mathrm{~h}$ prior to restoration, to assure the entire rehydration of dentinal tissue. Then, selected dentin areas were restored with the materials tested through the 
TABLE I. Materials Tested

\begin{tabular}{|c|c|c|c|c|c|c|}
\hline & Adhesive & Batch & Restorative & Batch & $\mathrm{n}$ & Manufacturer \\
\hline $\mathrm{SB} 1^{\mathrm{a}}$ & Scotchbond 1 & 19970515 & $\mathrm{Z} 100$ & 19990325 & 37 & 3M Dental Division Products, \\
\hline F2000 & F2000 Adhesive & 19990901 & F2000 compomer & 19990901 & 23 & St. Paul, MN \\
\hline OPTS & Optibond Solo & 902002 & Prodigy & 710331 & 32 & Kerr Corporation, Orange, CA \\
\hline
\end{tabular}

${ }^{\text {a }}$ Single Bond in U.S.

counterpart adjusted to the periphery of the area. Three adhesive restorative systems were tested in this study (Table I). As per manufacturer's instructions, dentin areas were conditioned before restoration with $37 \%$ ortophosphoric acid (Total Etch, 15 s), except in the specimens selected for F2000 adhesive, which has a self-etching primer containing $10 \%$ maleic acid.

Once the specimen were restored, the assembly formed by the restored test specimen and the counterpart was removed from the perfusion system, bolts were coupled to lids of the metal holders to allow traction, and the assembly was taken to a universal mechanical testing machine (Hounsfield HTI, Croydon, UK) and submitted to a traction test at a cross-head speed of $1 \mathrm{~mm} / \mathrm{min}$. Tensile bond strength (TBS) values were obtained (MPa) for each specimen. The bonded area (BA) was inspected under stereoscopic microscope to determine the type of fracture (adhesive, cohesive).

\section{STATISTICAL ANALYSIS}

To test the relationship between TBS and BA, first the best nonlinear curve estimation was determined (SPSS 9.0, SPSS Inc., Chicago, IL), trying linear, logarithmic, inverse, quadratic, cubic, compound, power, S Growth, exponential and logistic fittings for each material and for all three materials grouped.

\section{RESULTS}

Table II shows the area data measured in the three groups: small, medium, and large dentin areas. The resultant TBS values are shown in Table III, per area groups and per materials. In order to choose the best descriptive model, all the resultant models were inspected in each material. Attending to their adjusted $r$ coefficient, the best for Scotchbond 1 and for Optibond Solo was the inverse model. The best for F2000 was the power descriptive model, but the inverse model also had a very good fit in this case, so it is considered

TABLE II. Distribution of Areas $\left(\mathrm{mm}^{2}\right)$, per Groups

\begin{tabular}{llrrrr}
\hline Group & $n$ & \multicolumn{1}{c}{ Max } & \multicolumn{1}{c}{ Min } & mean & sd \\
\hline Small & 31 & 3.02 & 0.69 & 1.89 & 0.6 \\
Medium & 23 & 19.54 & 8.66 & 12.66 & 2.51 \\
Large & 38 & 110.86 & 58.91 & 83.63 & 13.78 \\
\hline
\end{tabular}

a good model for all materials. With all materials together the inverse model was also chosen. With the use of this model, the resulting formulas describe the relationship between BA and TBS per materials and also with all materials together, as can be seen in Table IV.

Figure 2 shows the fitting curve and its 95\% confidence intervals of the relationship between TBS and BA, when all materials are pooled together. The three different area groups can be seen clearly in this graph, which describes the increase in TBS when BA decreases, following an inverse relationship.

\section{DISCUSSION}

Regardless of the material tested, the results show that bond strength tends to increase as the bonding area decreases, a well-known fact. $^{8-9}$

The resin-dentin interface is a source of inherent defects that can propagate and act as stress raisers promoting interfacial failures, ${ }^{10}$ and the larger the bonded area of specimen,

TABLE III. TBS (MPa) Results per Area Groups and Materials

\begin{tabular}{llcccc}
\hline \multirow{2}{*}{ Material } & & \multicolumn{3}{c}{ Area Groups } & \\
\cline { 2 - 5 } SB1 & $n$ & Small & Medium & Large & Total \\
& Max. & 36.52 & 9.12 & 16 & 37 \\
& Min. & 0.39 & 2.01 & 7.78 & 36.52 \\
& Mean & 12.11 & 3.38 & 4.97 & 0.39 \\
& sd & 8.98 & 1.09 & 1.41 & 6.88 \\
OPTS & $n$ & 13 & 9 & 10 & 32 \\
& Max. & 22.81 & 7.24 & 7.35 & 22.81 \\
& Min. & 1.28 & 3.19 & 2.02 & 1.28 \\
& Mean & 9.43 & 5.51 & 4.46 & 6.78 \\
& sd & 5.94 & 1.52 & 1.7 & 4.5 \\
F2000 & $n$ & 6 & 5 & 12 & 23 \\
& Max. & 25.83 & 6.93 & 2.81 & 25.83 \\
& Min. & 4.91 & 4.47 & 1.03 & 1.03 \\
& Mean & 13.07 & 5.48 & 1.75 & 5.52 \\
& sd & 7.83 & 1.07 & 0.66 & 6.13 \\
Total & $n$ & 31 & 23 & 38 & 92 \\
& $n$ & 36.52 & 7.24 & 7.78 & 36.52 \\
& Max. & 0.39 & 2.01 & 1.03 & 0.39 \\
& Min. & 11.17 & 4.67 & 3.8 & 6.5 \\
& Mean & 7.5 & 1.61 & 1.91 & 5.66 \\
& sd & & & &
\end{tabular}


TABLE IV. Coefficients for the Inverse Model TBS $=\mathbf{C}+[\mathrm{B} / \mathrm{BA}]$, for Each and All Materials

\begin{tabular}{lcrc}
\hline & \multicolumn{2}{c}{ Coefficients } & \\
\cline { 2 - 3 } Material & $\mathrm{C}$ & $\mathrm{B}$ & ANOVA's Significance $p$ \\
\hline SB1 & 4.67 & 11.01 & $=0.004$ \\
OPTS & 4.65 & 7.44 & $=0.001$ \\
F2000 & 2.55 & 16.40 & $<0.0001$ \\
All materials & 4.17 & 10.35 & $<0.0001$ \\
\hline
\end{tabular}

the greater the probability of that area to contain one or more defects. ${ }^{10}$ This may be the explanation for failures of larger specimens submitted to traction tests at apparent low tensile bond strength.

In order to improve the stress distribution, in all specimens, except in the first group, a round cross-sectional shape at the adhesive interface was chosen. In the specimens of the first group, the entire exposed surface area was considered the testing area, both dentin and the surrounding enamel. Shapes of these areas were irregular, not perfectly round. In a previous study, ${ }^{8}$ cylindrical and rectangular cross-sectional specimens for microtensile tests were compared. The cylindrical shape distributed stresses along the interface better than the rectangular one did. The latter concentrated stresses at the corners and central area of the lateral aspects, instead of around the periphery of the bond surface.

Although the same cross-sectional shape and similar area sizes (in the two smaller area size groups tested) as those used by other authors ${ }^{8,10}$ were used, the tensile bond strength results were comparatively lower. It is assumed that hetero- geneity of dentin produces a significant effect on the bond strength, but this fact is not enough to explain the differences found between results in the present study and the ones obtained in previous works. So, it is necessary to analyze the reasons for these results.

First of all, the storage-media ( $70 \%$ ethanol solution) effect on bond strength is important. According to Goodis, Marshall, and White, ${ }^{11}$ the $70 \%$ ethanol solution resulted in the lowest fluctuations in permeability during their study, suggesting that the effect of time storage may stabilize the initial permeability changes, which after some point may no longer occur. But ethanol is a fixative agent for the protein component of dentine, causing tissue shrinkage and having a coagulant effect on proteins by displacing water, resulting in broken hydrogen bonds and collagen perturbation. ${ }^{12}$ This storage media could explain the degradation phenomena on dentine surfaces. Ethanol's effect on dentine substrates ${ }^{13}$ may then have an effect on results of etching pretreatment and/or priming, and a reduction in bonding strength may occur, due to a weak interface between the altered dentine substrates and the applied adhesive system.

All the specimens of the present study were submitted to perfusion ( $30 \mathrm{~cm} \mathrm{H}_{2} \mathrm{O}$ pressure), with distilled water used as perfusion fluid. Many authors have stated that bond strength of dentin adhesives is affected when dentin is maintained at physiological conditions, ${ }^{14-15}$ in most of the cases due to the wetness and to the type of fluid used. Level of pulpal pressure does not appear to have influence in dentin bond strengths. ${ }^{16,17}$ The presence of moist dentin seems to be a more important factor in adhesion because, according to Tao

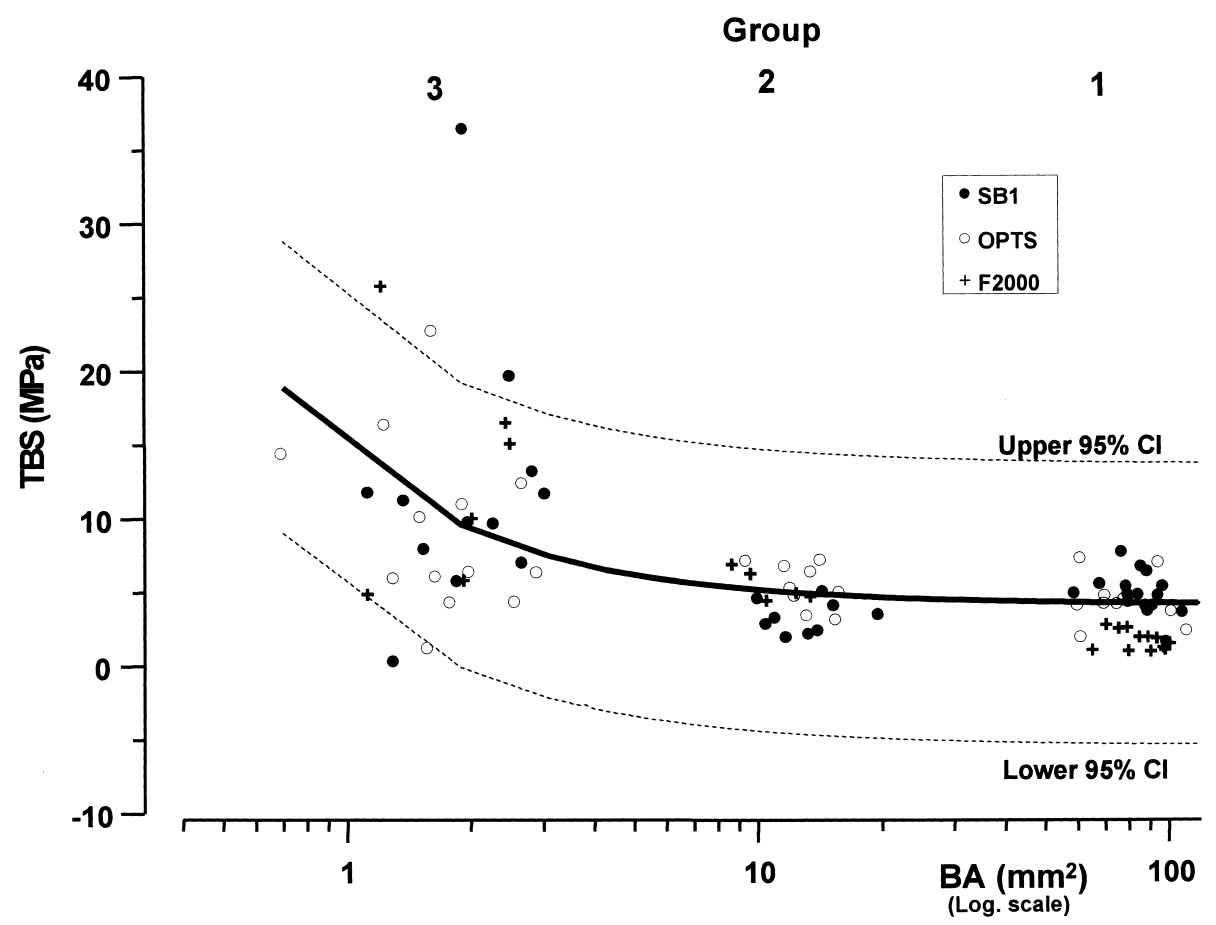

Figure 2. Fitting curve (and 95\% confidence intervals) of TBS versus BA. 1: large, 2: medium and 3: small areas. Note logarithmic scale in BA axis. 


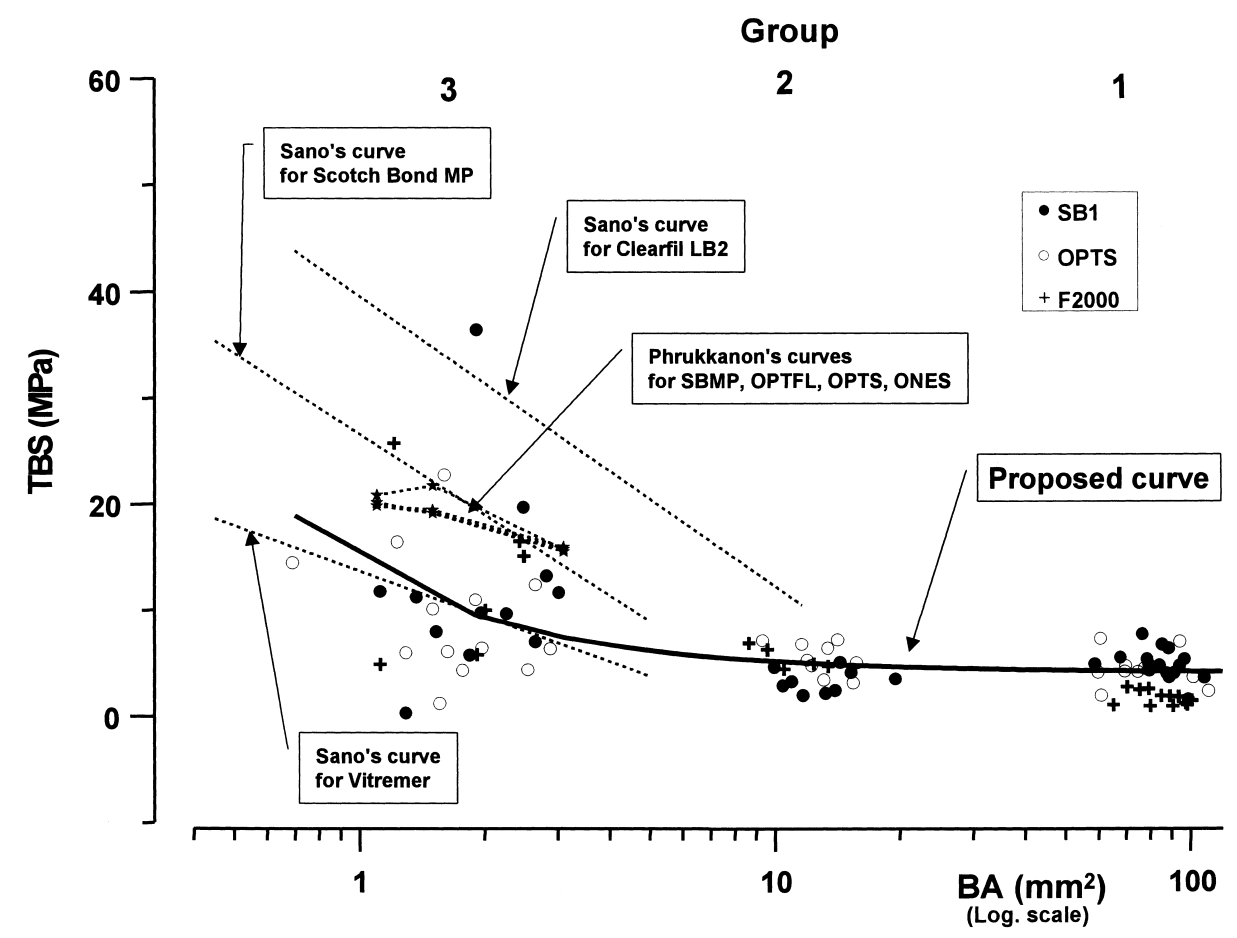

Figure 3. Proposed curve and previously published relationships. Note logarithmic scale in BA axis.

and Pashley, ${ }^{17}$ high bond strengths were produced on dentin that has low permeability, and low bond strengths were obtained on dentin that was highly permeable. Although new dentinal adhesives have been designed to perform properly on moist substrates, it is possible that excess water could interfere with the polymerization or the hybridized processes, thus affecting the final mechanical properties of interface. ${ }^{18,19}$ If the inverse relationship between dentin permeability and dentin bond strength is real, this fact could explain the low tensile bond strength values obtained here.

Composition of the perfusion fluid also plays an important role in dentin adhesion. A wide range of perfusing solutions, such as sterile phosphate-buffered saline ${ }^{16}$ physiologic saline, ${ }^{20,21}$ horse serum, ${ }^{23}$ bovine serum, ${ }^{24}$ and protein solutions, ${ }^{22}$ have been tested in different studies in attempts to reproduce the dentinal fluid in vitro. But little is known about the exact composition of this dentinal fluid, although seems to be that albumin and globulins are the main components. ${ }^{23}$ The presence of proteins in the perfusing solution may cause a precipitation within the tubules, blocking them and reducing the dentin permeability. ${ }^{23}-25$ This coagulation effect is not likely to occur when distilled water is used, as in this study.

Despite these differences, the present results indicate that the size of the adhesive area highly influences the mechanical resistance of the interface between adhesive and dentin substrate, with an inverse relationship between both parameters. The fitting curve obtained from the tensile bond strength data shows it clearly. Larger areas are correlated with lower resistance values, as predicted in Griffith's theory, ${ }^{5}$ a fact that was mentioned above. So, this is an expected relationship, and is in agreement with the findings of Sano et al. ${ }^{6}$ and Phrukkanon, Burrow, and Tyas, ${ }^{8}$ who in their previous works established the importance of the area size related to the tensile tests. The first used areas ranging from 0.25 to 11.65 $\mathrm{mm}^{2}$ and other methodology and the second from 1.1 to 3.1 $\mathrm{mm}^{2}$. The present study employs areas ranging from 0.69 to $110.86 \mathrm{~mm}^{2}$, a range much wider than these works. Furthermore, the equations of the regression lines relating tensile bond strength to the bonded surface area obtained are different from those obtained here. In none of these works were perfused specimens used, which can help to explain the differences in magnitude of TBS.

As can be seen in Figure 3, both predictions fit with the present data. However, both of them predict negative TBS for larger areas (circa $100 \mathrm{~mm}^{2}$ ). The present formula has, then, the advantage of considering larger bonded areas as would appear, for example, in bonded laminate veneers, while fitting also to smaller ones, as in laboratory testing.

Certainly, there are two cases where, despite the smallest BA being used, TBS was very low. These two cases (one SB1 and one OPTS) were included in calculations because their TBS result was greater than zero, although there is a reasonable option to exclude both cases. If they were excluded from the series, the new formulation of the model would be TBS = $4.10+11.77 / \mathrm{BA}$ with an adjusted $r^{2}=0.36$. Its representation does not differ much from the previous one.

Due to the design of the cavities used in the second and third group, there is another factor to be analyzed. Although the cavities through the amalgam layer were designed as nonretentive, the presence of walls could cause some friction when the restorative material was submitted to traction test. 
Therefore, the tensile bond strength results could be lower than the obtained ones if the bonded area used is a completely flat surface instead of a cavity. Another factor related to cavity's design is the presence of the bonded amalgam layer, which was placed to frame a concrete study area. Based in the reports of Prati et al., ${ }^{24}$ Scotchbond Multipurpose Plus (with prime and adhesive being the same as that in Scotchbond Multipurpose System) creates a hybrid layer between 2 and 7 $\mu \mathrm{m}$. Although cavities reached a mean depth of $250 \mu \mathrm{m}$, enough to remove the hybrid layer created between dentin and amalgam, some resin tags could remain within the tubules, preventing complete resin infiltration, and hence weakening the bond. Moreover, in both medium and small groups the area used to calculate TBS is the projection of the real area where the materials were tested. This will probably result in a negligible difference.

The conclusion of this study, despite the technique problems, is that it confirmed that there is a constant and inverse relationship between tested area size and tensile bond strength.

\section{REFERENCES}

1. Lin C, Douglas WH. Failure mechanisms at the human dentinresin interface: A fracture mechanics approach. J Biomech 1994;27:1037-1047.

2. Lin C, Chen R, Liu C, Lian S. Failure criteria of dentin-resin tensile bond test-A fracture mechanics approach. Scr Mater 1998;38:115-121.

3. Sudsangiam S, Van Noort R. Do dentin bond strength tests serve a useful purpose?. J Adhesive Dent 1999;1:57-67.

4. May KN, Swift EJ, Bayne SC. Bond strengths of a new dentin adhesive system. Am J Dent 1997;10:195-198.

5. Griffith AA. The phenomena of rupture and flow in solids. Philos Trans R Soc London 1920;A221:168-198.

6. Sano H, Shono T, Sonoda H, Takatsu T, Ciucchi B, Carvalho R, Pashley DH. Relationship between surface area for adhesion and tensile bond strength-Evaluation of a micro-tensile bond test. Dent Mater 1994;10:236-240.

7. Van Noort R, Cardew GE, Howard IC, Noroozi S. The effect of local interfacial geometry on the measurement of the tensile bond strength to dentin. J Dent Res 1989;70:889-893.

8. Phrukkanon S, Burrow MF, Tyas MJ. The influence of crosssectional shape and surface area on the microtensile bond test. Dent Mater 1998;14:212-221.

9. Cardoso PEC, Braga RR, Carrilho MRO. Evaluation of microtensile, shear and tensile tests determining the bond strength of three adhesive systems. Dent Mater 1998;14:394-398.
10. Phrukkanon S, Burrow MF, Tyas MJ. Effect of cross-sectional surface area on bond strengths between resin and dentin. Dent Mater 1998;14:120-128.

11. Shono Y, Terashita M, Pashley EL, Brewer PD, Pashley DH. Effects of cross-sectional area on resin-enamel tensile bond strength. Dent Mater 1997; 13: 290-296.

12. Toparli M, Aksoy T. Fracture toughness determination of composite resin and dentin/composite resin adhesive interfaces by laboratory testing and finite element models. Dent Mater 1998; 14:287-293.

13. Goodis HE, Marshall GW, White JM. The effects of storage after extraction of the teeth on human dentine permeability in vitro. Arch Oral Biol 1991;36:561-566.

14. Kiernan JA. Histological and histochemical methods. Theory and practice (1st ed.). Oxford: Pergamon Press; 1981. p 8.

15. Lee SY, Lin CT. Storage effects on dentine structure and on resultant composite bond strengths. J Oral Rehab 1997;24:823834.

16. Mitchem JC, Terkla LG, Gronas DG. Bonding of resin dentin adhesives under simulated physiological conditions. Dent Mater 1988;4:351-353.

17. Tao L, Pashley DH. The relationship between dentin bond strengths and dentin permeability. Dent Mater 1989;5:133-139.

18. Tagami J, Tao L, Pashley DH. Correlation among dentin depth, permeability, and bond strength of adhesive resins. Dent Mater 1990;6:45-50.

19. Prati C, Simpson M, Mitchem J, Tao L, Pashley DH. Relationship between bond strength and microleakage measured in the same Class I restorations. Dent Mater 1992;8:37-41.

20. Pameijer CH, Louw NP. Significance of pulpal pressure during clinical bonding procedures. Am J Dent 1997;10:214-218.

21. Del-Nero MO, Escribano N, De La Macorra JC. Analysis of sealing vs tensile bond strength of eight adhesive restorative material systems. J Adhesive Dent 2000;2:117-127.

22. Jacobsen T, Söderholm KJ. Some effects of water on dentin bonding. Dent Mater 1995;11: 132-136.

23. Augustin C, Paul SJ, Lüthy H, Schärer P. Perfusing dentine with horse serum or physiologic saline: Its effect on adhesion of dentine bonding agents. J Oral Rehab 1998;25:596-602.

24. Nikaido T, Burrow MF, Tagami J, Takatsu T. Effect of pulpal pressure on adhesion of resin composite to dentin: Bovine serum versus saline. Quintessence Int 1995;26:221-226.

25. Hahn C, Overton B. The effects of immunoglobulins on the convective permeability of human dentine in vitro. Arch Oral Biol 1997;42:835-843.

26. Knutsson G, Jontell M, Bergenholtz G. Determination of plasma proteins in dentinal fluid from cavities prepared in healthy young human teeth. Arch Oral Biol 1994;39:185.

27. Prati C, Chersoni S, Mongiorgi R, Pashley DH. Resin-infiltrated dentin layer formation of new bonding systems. Oper Dent 1998;23:185-194. 